\title{
Dynamical response of a Timoshenko beams on periodical nonlinear supports subjected to moving forces
}

\author{
T. Hoang ${ }^{\mathrm{a}, *}$, D. Duhamel ${ }^{\mathrm{a}}$, G. Foret ${ }^{\mathrm{a}}$ \\ ${ }^{a}$ Université Paris-Est, Navier (UMR 8205 ENPC-IFSTTAR-CNRS), Ecole Nationale des \\ Ponts et Chaussées, 6 et 8 Avenue Blaise Pascal, Cité Descartes, Champs-sur-Marne, 77455 \\ Marne-la-Vallée Cedex 2, France
}

\begin{abstract}
The periodically supported Timoshenko beam subjected to moving forces has been investigated by numerous researches. The existed models have been developed for linear supports, and this article presents a new one for nonlinear supports. By using a periodic condition and the Fourier series development, the dynamic equation of the Timoshenko beam leads to a relation between the beam displacements and the reaction forces of the supports. This relation does not depend on the support behavior and it exists also for the Euler-Bernoulli beam. Then, the responses can be obtained by combining this relation and the constitutive law of the supports. A numerical method based on discretization of the time and frequency responses has been developed for nonlinear supports. Moreover, the influence of the beam model has been studied with numerical examples of linear and nonlinear supports. The results show that the Timoshenko beam should be used for the moving forces with high speed and/or the supports with large stiffness.
\end{abstract}

Keywords: Periodically supported beam, System equivalence, Non-linear, Dynamics, Fourier transform, Timoshenko beam

\footnotetext{
*Corresponding author

Email address: tien.hoang@enpc.fr (T. Hoang)
} 


\section{Introduction}

The periodically supported beam subjected to moving forces has been investigated in numerous publications [1 10]. In these articles, the Euler-Bernoulli or Timoshenko beams resting on identical supports at periodical intervals have 5 been considered in steady-state. The response to moving forces are calculated analytically when the supports are linear. However, these models cannot be extended easily for nonlinear supports. Recently, the dynamics of a periodically supported beam has been represented by the system equivalence [11] by using a periodic condition of reaction forces. This model could work for nonlinear supports, but the author has not presented a method to compute the dynamical responses. Some other researches have considered the model of beams on nonlinear foundations (i.e. the beam is supported continuously) by using the perturbation technique [12 14], the Galerkin method [15] or the numerical methods 16, 17].

This article presents a complete analytic model for the dynamics of beams resting on periodic nonlinear supports. A relation between the beam displacement and the reactions forces has been established from the periodic condition and the dynamic equation of the Timoshenko beam. Then, a numerical method has been developed to compute the response form this relation and the constitutive law of the nonlinear supports. Moreover, a comparison between the Timoshenko and Euler-Bernoulli beam models has been performed with numerical examples of linear and nonlinear behaviors.

\section{Periodically supported Timoshenko beam}

\subsection{Dynamical equations in steady-state}

25

Let's consider an infinite Timoshenko beam resting on identical supports at periodical intervals as shown in Figure 1 The beam is subjected to the moving forces $Q_{j}$ characterized by the distance to the first force $D_{j}$. Let $R_{n}(t)$ be the reaction force of a support at the coordinate $x=n l$ (with $n \in \mathbb{Z}$ ). 


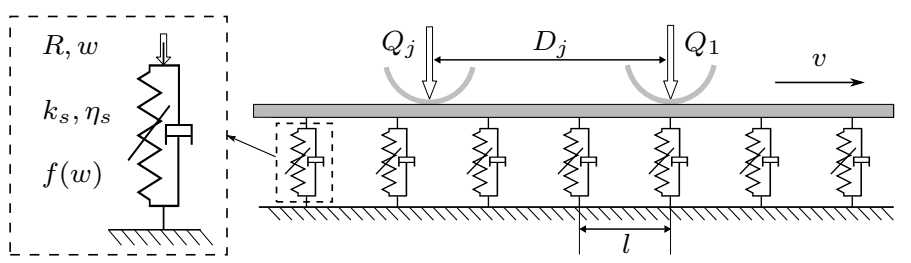

Figure 1: Forces applied on a periodically supported beam

In steady-state, we suppose that all supports are equivalent and their responses are described by the same function, but with a delay which equals to the time for the forces to cover the distance between them. In other words, the reaction force repeats when the moving forces pass from one support to another

$$
R_{n}(t)=R\left(t-\frac{n l}{v}\right)
$$

where $R(t)$ is the reaction force of the support at the origin of the reference system $x=0$. The total force applied on the beam can be represented with the help of the Dirac functions

$$
F(x, t)=\sum_{n=-\infty}^{\infty} R\left(t-\frac{x}{v}\right) \delta(x-n l)-\sum_{j=1}^{K} Q_{j} \delta\left(x+D_{j}-v t\right)
$$

In addition, we have the dynamic equations of the Timoshenko beam

$$
\left\{\begin{array}{l}
\rho S \frac{\partial^{2} w_{r}}{\partial t^{2}}=\kappa S G\left(\frac{\partial^{2} w_{r}}{\partial x^{2}}-\frac{\partial \phi_{r}}{\partial x}\right)+F(x, t) \\
\rho I \frac{\partial^{2} \phi_{r}}{\partial t^{2}}=E I \frac{\partial^{2} \phi_{r}}{\partial x^{2}}+\kappa S G\left(\frac{\partial w_{r}}{\partial x}-\phi_{r}\right)
\end{array}\right.
$$

where $w_{r}, \phi_{r}$ are the displacement and the rotation of the section of the beam; 30 $\rho, E$ are the density, the Young's modulus and $S, I, \kappa, G$ are the section, the inertia, the shear coefficient and the shear modulus of the beam.

Equations (2) and (3) define the dynamics of the beam and its supports in the steady-state. Thereafter, we will resolve these equations by performing a Fourier transform with regard to the time $t$, and then using the Fourier series development with regard to $x$. Let's denote $\partial_{t}, \partial_{x}$ the derivations with regard to $t$ and $x$. By performing the Fourier transform of equation (3) with regard to 
$t$, we obtain

$$
\left\{\begin{array}{l}
\kappa S G \partial_{x} \hat{\phi}_{r}=\kappa S G \partial_{x}^{2} \hat{w}_{r}+\rho S \omega^{2} \hat{w}_{r}+\hat{F} \\
-\kappa S G \partial_{x} \hat{w}_{r}=E I \partial_{x}^{2} \hat{\phi}_{r}-\left(\kappa S G-\rho I \omega^{2}\right) \hat{\phi}_{r}
\end{array}\right.
$$

where the hat stands for the Fourier transform with regard to $t$. Particularly, we obtain the following result from equation (2)

$$
\hat{F}=\mathrm{e}^{-\frac{\mathrm{i} \omega}{v} x}\left(\hat{R}(\omega) \sum_{n=-\infty}^{\infty} \delta(x-n l)-\sum_{j=1}^{K} \frac{Q_{j}}{v} \mathrm{e}^{-\frac{\mathrm{i} \omega}{v} D_{j}}\right)
$$

Thus, $\mathrm{e}^{\mathrm{i} \omega} x \hat{F}$ is periodic with regard to $x$. Therefore, if we put

$$
\hat{w}_{r}=\Psi(x, \omega) \mathrm{e}^{-\frac{\mathrm{i} \omega}{v} x} \quad \text { and } \quad \hat{\phi}_{r}=\Phi(x, \omega) \mathrm{e}^{-\frac{\mathrm{i} \omega}{v} x}
$$

equation (4) becomes

$$
\left\{\begin{array}{l}
\kappa S G\left(\partial_{x} \Phi-\frac{\mathrm{i} \omega}{v} \Phi\right)=\kappa S G\left(\partial_{x}^{2} \Psi-2 \frac{\mathrm{i} \omega}{v} \partial_{x} \Psi-\frac{\omega^{2}}{v^{2}} \Psi\right)+\rho S \omega^{2} \Psi+\mathrm{e}^{\frac{\mathrm{i} \omega}{v} x} \hat{F} \\
\kappa S G\left(\frac{\mathrm{i} \omega}{v} \Psi-\partial_{x} \Psi\right)=E I\left(\partial_{x}^{2} \Phi-2 \frac{\mathrm{i} \omega}{v} \partial_{x} \Phi-\frac{\omega^{2}}{v^{2}} \Phi\right)-\left(\kappa S G-\rho I \omega^{2}\right) \Phi
\end{array}\right.
$$

By the Floquet's theorem [18], equation (7) has a periodic solution. We can find this solution by using the Fourier series developments of $\Phi$ and $\Psi$ (see Appendix A). Thereafter, by combining the results of $\Psi$ and $\Phi$ with equation (6) we obtain

$$
\left\{\begin{array}{l}
\hat{w}_{r}(x, \omega)=\hat{R}(\omega) \sum_{n=-\infty}^{\infty} \tilde{p}_{n} \mathrm{e}^{\mathrm{i} x\left(\frac{2 \pi n}{l}-\frac{\omega}{v}\right)}-\tilde{p}_{0} \frac{l}{v} \sum_{j=1}^{K} Q_{j} \mathrm{e}^{-\frac{\mathrm{i} \omega}{v}\left(x+D_{j}\right)} \\
\hat{\phi}_{r}(x, \omega)=\hat{R}(\omega) \sum_{n=-\infty}^{\infty} \tilde{q}_{n} \mathrm{e}^{\mathrm{i} x\left(\frac{2 \pi n}{l}-\frac{\omega}{v}\right)}-\tilde{q}_{0} \frac{l}{v} \sum_{j=1}^{K} Q_{j} \mathrm{e}^{-\frac{\mathrm{i} \omega}{v}\left(x+D_{j}\right)}
\end{array}\right.
$$

where $\tilde{p}_{n}, \tilde{q}_{n}(n \in \mathbb{Z})$ are the Fourier coefficients of $\Psi, \Phi$ calculated by equation (A7) in Appendix A. We can reduce equation (8) by defining $\eta(x, \omega), \gamma(x, \omega)$ as follows

$$
\left\{\begin{array}{l}
\eta(x, \omega)=\sum_{n=-\infty}^{\infty} \tilde{p}_{n} \mathrm{e}^{\mathrm{i} x\left(\frac{2 \pi n}{l}-\frac{\omega}{v}\right)} \\
\gamma(x, \omega)=\sum_{n=-\infty}^{\infty} \tilde{q}_{n} \mathrm{e}^{\mathrm{i} x\left(\frac{2 \pi n}{l}-\frac{\omega}{v}\right)}
\end{array}\right.
$$


Indeed, $\eta, \gamma$ in the last equation can be reduced to simple analytical functions as shown in equations (B11) and (B14) of Appendix B. Then, by substituting equation (9) into equation (8), we obtain

$$
\left\{\begin{array}{l}
\hat{w}_{r}(x, \omega)=\hat{R}(\omega) \eta(x, \omega)-\tilde{p}_{0} \frac{l}{v} \sum_{j=1}^{K} Q_{j} \mathrm{e}^{-\frac{\mathrm{i} \omega}{v}\left(x+D_{j}\right)} \\
\hat{\phi}_{r}(x, \omega)=\hat{R}(\omega) \gamma(x, \omega)-\tilde{q}_{0} \frac{l}{v} \sum_{j=1}^{K} Q_{j} \mathrm{e}^{-\frac{\mathrm{i} \omega}{v}\left(x+D_{j}\right)}
\end{array}\right.
$$

Equation (10) is a simple relation between the Fourier transforms of the beam displacement and the reaction force. This is a result of the periodicity condition and the dynamic equation of the Timoshenko beam, which do not depend on the support behaviour. Once the reaction force is calculated, this equation can be used to compute the response of the beam. In the next section, we will introduce a system equivalence based on this relation.

\subsection{System equivalence}

In order to calculate the reaction force of the support, we need to compute the displacement of the beam at the support position $w(t)=w_{r}(0, t)$, or its Fourier transform $\hat{w}(\omega)=\hat{w}_{r}(0, \omega)$. By substituting $x=0$ into equation (10), we have

$$
\hat{w}_{r}(0, \omega)=\hat{R}(\omega) \eta(0, \omega)-\tilde{p}_{0} \frac{l}{v} \sum_{j} Q_{j} e^{-i \frac{\omega}{v} D_{j}}
$$

Hence, we can also write

$$
\hat{R}(\omega)=\mathcal{K}_{T} \hat{w}(\omega)+\mathcal{Q}_{T}
$$

where $\mathcal{K}_{T}$ and $\mathcal{Q}_{T}$ are defined by

$$
\mathcal{K}_{T}=\eta^{-1}(0, \omega) \quad \text { and } \quad \mathcal{Q}_{T}=\mathcal{K}_{T} \tilde{p}_{0} \frac{l}{v} \sum_{j=1}^{K} Q_{j} e^{-i \frac{\omega}{v} D_{j}}
$$

with $\tilde{p}_{0}(\omega), \eta(0, \omega)$ are calculated by equations (A10) and (B15) in the appendices.

Equation (12) is a linear relation between the force and the displacement applied on the support at $x=0$, and it holds for all supports because of the 
periodicity condition. This relation is the same as the constitutive law of an equivalent spring with stiffness $\mathcal{K}_{T}$ and pre-force $\mathcal{Q}_{T}$. Therefore, we call the system equivalence of a periodically supported Timoshenko beam, which existed also for Euler-Bernoulli beams (see 11]). Equation (12) explains the distribution mechanism of the moving forces $Q_{j}$ to the supports via the beam. When a moving force comes toward and leaves away the support along the direction of the beam, the reaction force of the support increases and decreases respectively. This process is the same as a force applied on the support via the equivalent spring.

The comparison of the system equivalences with two parameters stiffness and pre-force for Euler-Bernoulli and Timoshenko beams is shown in Table 1 . We see that the stiffness $\mathcal{K}$ depends on two parameters $\lambda_{1,2}$ and $C_{1,2}$ which are different between the two beam models. However, if the shear modulus $\kappa G$ and the ratio $E / \rho$ tend to infinity, $\lambda_{1,2}$ and $C_{1,2}$ of the Timoshenko beam tend to the ones of the Euler-Bernoulli beam. Therefore, the stiffness and pre-force for the two beam models are equivalent when the Timoshenko beam does not include the shear modulus $\kappa G$ and the ratio $E / \rho$. This phenomenon agrees well with the beam theories.

Table 1: System equivalence for Euler-Bernoulli and Timoshenko beams

$$
\begin{aligned}
& \hat{R}(\omega)=\mathcal{K}(\omega) \hat{w}(\omega)+\mathcal{Q}(\omega) \\
& \mathcal{Q}(\omega)=\mathcal{K}(\omega) \frac{\tilde{p}_{0} l}{v} \sum_{j=1}^{K} Q_{j} \mathrm{e}^{-\mathrm{i} \omega \frac{D_{j}}{v}} \\
& \mathcal{K}(\omega)=2 E I\left(\lambda_{1}^{2}+\lambda_{2}^{2}\right)\left(\frac{C_{1}}{\lambda_{1}} \frac{\sin l \lambda_{1}}{\cos l \lambda_{1}-\cos \frac{l \omega}{v}}-\frac{C_{2}}{\lambda_{2}} \frac{\sinh l \lambda_{2}}{\cosh l \lambda_{2}-\cos \frac{l \omega}{v}}\right)^{-1}
\end{aligned}
$$

\begin{tabular}{l|l}
\hline Euler - Bernoulli beam & Timoshenko beam \\
$\lambda_{1,2}^{2}=\sqrt{\frac{\rho S \omega^{2}}{E I}}$ & $\lambda_{1,2}^{2}=\sqrt{\frac{\rho S \omega^{2}}{E I}+\frac{\omega^{4}}{4}\left(\frac{\rho}{E}-\frac{\rho}{\kappa G}\right)^{2}} \pm \frac{\omega^{2}}{2}\left(\frac{\rho}{E}+\frac{\rho}{\kappa G}\right)$ \\
$C_{1,2}=1$ & $C_{1,2}=1-\frac{\rho I \omega^{2} \mp E I \lambda_{1,2}^{2}}{\kappa S G}$ \\
$\tilde{p}_{0} l=\frac{1}{E I \frac{\omega^{4}}{v^{4}}-\rho S \omega^{2}}$ & $\tilde{p}_{0} l=\frac{\kappa S G-\rho I \omega^{2}+E I \frac{\omega^{2}}{v^{2}}}{\kappa S G\left(E I \frac{\omega^{4}}{v^{4}}-\rho S \omega^{2}\right)-\rho S I\left(\kappa G+E-\rho v^{2}\right) \frac{\omega^{4}}{v^{2}}}$ \\
\hline
\end{tabular}




\begin{tabular}{lclc}
\multicolumn{4}{l}{ Table 2: Parameters of a periodically supported beam } \\
\hline Mass density & $\rho$ & $\mathrm{kg} / \mathrm{m}^{3}$ & 7850 \\
Young's modulus & $E$ & $\mathrm{GPa}$ & 210 \\
Shear modulus & $G$ & $\mathrm{GPa}$ & 81 \\
Shear coefficient & $\kappa$ & & 0.4 \\
Section & $S$ & $\mathrm{~m}^{2}$ & $7.69 \mathrm{e}-3$ \\
Flexion inertia & $I$ & $\mathrm{~m}^{4}$ & $3.055 \mathrm{e}-5$ \\
Sleeper spacing & $l$ & $\mathrm{~m}$ & 0.6 \\
Moving force & $Q$ & $\mathrm{kN}$ & 100 \\
Speed of moving force & $v$ & $\mathrm{~km} / \mathrm{h}$ & 300 \\
\hline
\end{tabular}

Example

Figures 2 and 3 show an example of the stiffness and the pre-force for the Timoshenko and the Euler-Bernoulli beams with the parameters presented in Table 2 We see that the two beam models give almost the same equivalent preforce. Otherwise, the Timoshenko beam gives a smaller equivalent stiffness than the Euler-Bernoulli beam. It is remarkable that the difference takes place only at the maximum peaks of the stiffness which correspond to high frequencies. In other words, the influence of the beam models is more important at high

frequencies. In the next sections, we will calculate the responses for two beam models with linear and nonlinear support behaviours in order to compare the two beam models.

\section{Calculation of responses}

\subsection{Linear supports}

Let's consider the viscoelastic supports represented by a stiffness $k_{s}$ and a damping coefficient $\eta_{s}$. The dynamical stiffness of the supports is $K_{s}=k_{s}+\mathrm{i} \omega \eta_{s}$ and their constitutive law is given by

$$
\hat{R}(\omega)=-K_{s} \hat{w}(\omega)
$$




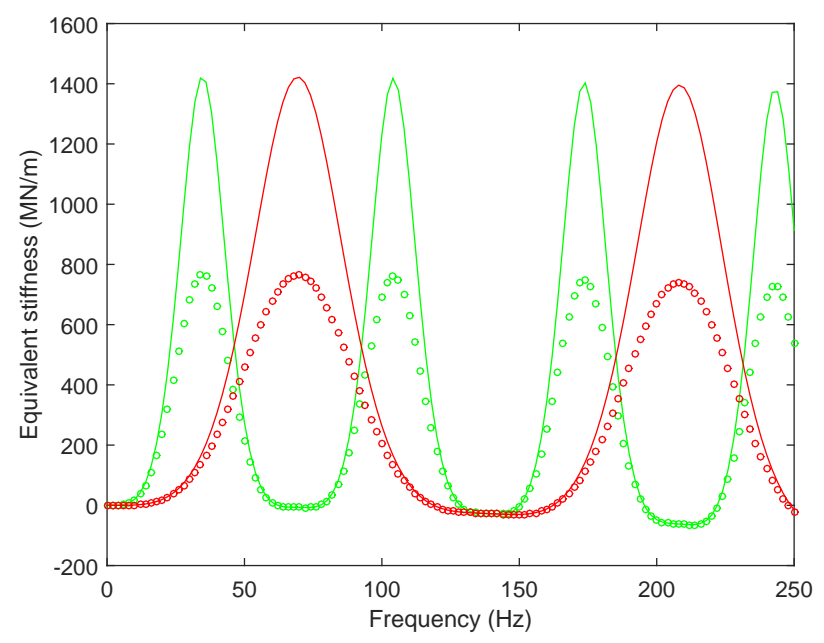

Figure 2: Equivalent stiffness of Timoshenko (circle) and Euler-Bernoulli (continuous line) beams with moving velocity $150 \mathrm{~km} / \mathrm{h}$ (green) and $300 \mathrm{~km} / \mathrm{h}$ (red)

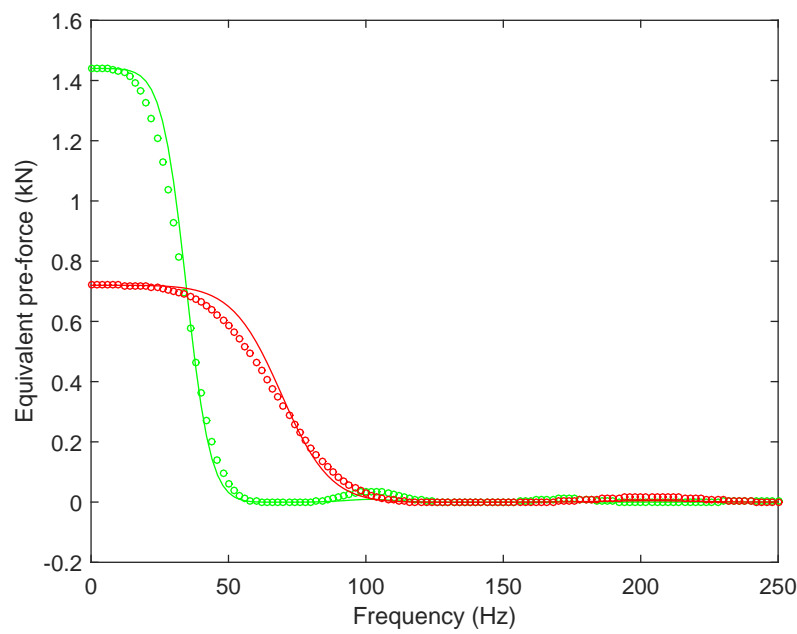

Figure 3: Equivalent pre-force of Timoshenko (circle) and Euler-Bernoulli (continuous line) beams with moving velocity $150 \mathrm{~km} / \mathrm{h}$ (green) and $300 \mathrm{~km} / \mathrm{h}$ (red)

By combining equations (12) and (14), we obtains the response of the supports

$$
\left\{\begin{array}{l}
\hat{R}(\omega)=\frac{K_{s} \mathcal{Q}_{T}}{\mathcal{K}_{T}+K_{s}} \\
\hat{w}_{s}(\omega)=\frac{-\mathcal{Q}_{T}}{\mathcal{K}_{T}+K_{s}}
\end{array}\right.
$$


Then, by substituting the last results and equation (13) into equation (10) we obtain the response of the beam

$$
\left\{\begin{array}{l}
\hat{w}_{r}(x, \omega)=\mathcal{Q}_{T}\left(\frac{K_{s} \eta(x, \omega)}{K_{s}+\mathcal{K}_{T}}-\eta(0, \omega) \mathrm{e}^{-\mathrm{i} \frac{\omega}{v} x}\right) \\
\hat{\phi}_{r}(x, \omega)=\mathcal{Q}_{T}\left(\frac{K_{s} \gamma(x, \omega)}{K_{s}+\mathcal{K}_{T}}-\gamma(0, \omega) \mathrm{e}^{-\mathrm{i} \frac{\omega}{v} x}\right)
\end{array}\right.
$$

Equations (15) and (16) describe completely the responses in the frequency domain of the supports and the beam. The real-time responses can be obtained by performing the inverse Fourier transforms.

\section{Example}
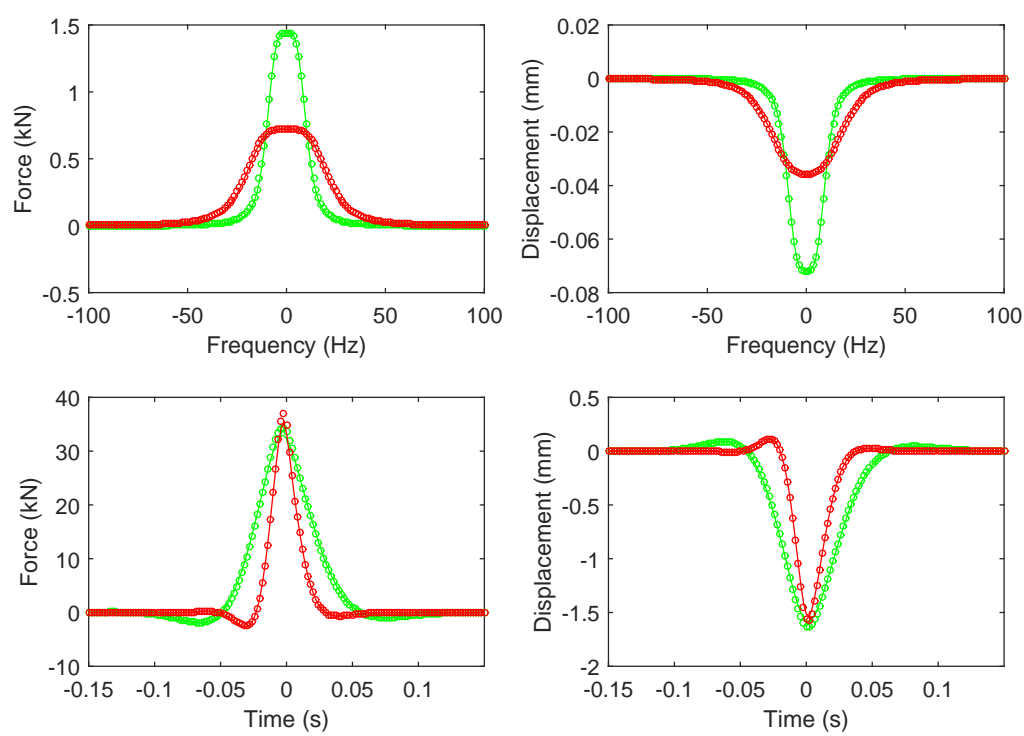

Figure 4: Responses of the supports with Timoshenko (circle) and Euler-Bernoulli (continuous line) beams with a moving velocity $150 \mathrm{~km} / \mathrm{h}$ (green) and $300 \mathrm{~km} / \mathrm{h}$ (red)

The responses have been calculated for the linear support by using equation

(16) with the beam parameters given in table 2 and the support parameters $k_{s}=20 \mathrm{MN} / \mathrm{m}, \eta_{s}=0.1 \mathrm{MNs} / \mathrm{m}$. Figure 4 shows the reaction force and the displacement of the support in the frequency domain and the time domain. Although the equivalent stiffness of the two beam models are different, the 
responses are almost the same because they are affected more by the lower frequencies. Moreover, the larger velocity leads to responses of smaller amplitudes, but of greater frequency range. Next, we will investigate the influence of the beam model on the responses by parametric studies.

\section{Influence of beam models}

The Timoshenko beam theory shows that the two beam models can be equivto the previous example is 0.0715 , which is closed to the limit of this condition. Therefore, it is necessary to study the influence of the beam model on the dynamic responses. The external parameters which could affect on the responses are the moving force speed and the stiffness of the support. Figures 5 and 6
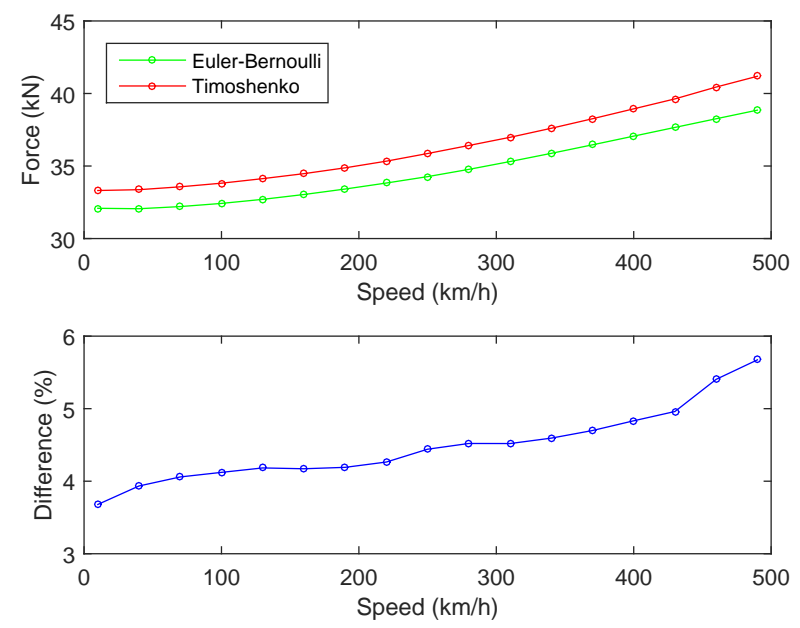

Figure 5: Influence of the moving force speed on the reaction forces

show the maximum of the reaction force versus these parameters. When the moving force speed or the stiffness of the support increase, the reaction forces increase, and the difference between the two beam models is more important. These results show that the Timoshenko beam model should be used for moving forces of high speed or supports of large stiffness. 

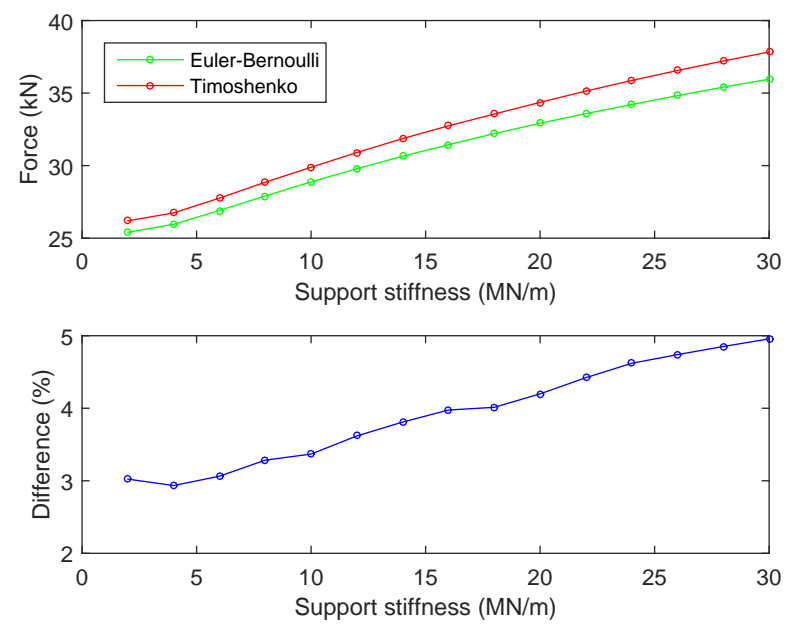

Figure 6: Influence of the support stiffness on the reaction forces

\subsection{Nonlinear supports}

Now we consider a system of supports that has a nonlinear behaviour as shown in figure 1 The reaction force of a support is given by

$$
R(t)=-\left(k_{s} w+\eta_{s} w^{\prime}+f(w)\right)
$$

Combining equations (12) and (17) gives a system of two equations with two unknowns $R$ and $w$. In order to simplify the linear part of the constitutive law, we put

$$
P(t)=R+k_{s} w+\eta_{s} w^{\prime}
$$

By performing the Fourier transform, we can also write as follows

$$
\hat{P}(\omega)=\hat{R}+K_{s} \hat{w}
$$

where $K_{s}=k_{s}+i \omega \eta_{s}$. By combining equations (19) and (18) with equations (12) and (17) respectively, we obtain

$$
\left\{\begin{array}{l}
P=-f(w) \\
\hat{P}=\left(\mathcal{K}_{T}+K_{s}\right) \hat{w}+\mathcal{Q}_{T}
\end{array}\right.
$$


When $f(w)=0$, the last equation is exactly the same result as for linear supports presented in equation (15). When $f(w) \neq 0$, we got one equation in the frequency domain and the other in the time domain. To resolve this problem, we will discretize the unknown functions $P(t)$ and $w(t)$ and their Fourier transforms. It is remarkable that these functions (and their Fourier transforms) have bounded supports (see the numerical examples in section 3.1). Therefore, we can use the Shannon sampling theorem [19] to discretize these functions.

\section{Numerical method}

Suppose that it exists $t_{\max }, \omega_{\max }$ provided that

$$
\begin{cases}P(t) \simeq 0, w(t) \simeq 0 & \text { if }|t| \geq t_{\max } \\ \hat{P}(\omega) \simeq 0, \hat{w}(\omega) \simeq 0 & \text { if }|\omega| \geq \omega_{\max }\end{cases}
$$

We discretize the responses $w(t), P(t)$ and their Fourier transforms by considering their values at $t=n T$ and $\omega=n \Omega$ with $|n| \leq N$. The discretization has to satisfy the Shannon sampling theorem [19]

$$
\omega_{\max } \geq \frac{\pi}{T} \text { and } t_{\max } \geq \frac{\pi}{\Omega}
$$

Because $t_{\max }=N T$ and $\omega_{\max }=N \Omega$, the last condition becomes

$$
N T \Omega \geq \pi
$$

The discretization of the first equation of (20) becomes

$$
P_{n}=-f\left(w_{n}\right) \quad|n| \leq N
$$

where $P_{n}=P(n T)$ and $w_{n}=w(n T)$. In order to discretize the second equation of (201), we need to compute the Fourier transforms of the discretized responses $P_{n}$ and $w_{n}$, which are given by (see [19])

$$
\begin{cases}\hat{P}_{d}(\omega)=\sum_{n} P_{n} \mathrm{e}^{-\mathrm{i} n T \omega}=\frac{\hat{P}(\omega)}{T} & |\omega| \leq \frac{\pi}{T} \\ \hat{w}_{d}(\omega)=\sum_{n} w_{n} \mathrm{e}^{-\mathrm{i} n T \omega}=\frac{\hat{w}(\omega)}{T} & |\omega| \leq \frac{\pi}{T}\end{cases}
$$


Therefore, the discretization of the Fourier transforms $(\hat{P}, \hat{w})$ is deduced from the last equation

$$
\begin{cases}\hat{P}_{m}=\hat{P}(m \Omega)=T \sum_{n} P_{n} \mathrm{e}^{-\mathrm{i} n m T \Omega} & |m \Omega| \leq \frac{\pi}{T} \\ \hat{w}_{m}=\hat{w}(m \Omega)=T \sum_{n} w_{n} \mathrm{e}^{-\mathrm{i} n m T \Omega} & |m \Omega| \leq \frac{\pi}{T}\end{cases}
$$

By combining the conditions of equations (23), (24) and the last equation, we obtain

$$
N \Omega T=\pi
$$

and we have

$$
\begin{cases}\hat{P}_{m}=T \sum_{n=-N}^{N} P_{n} \mathrm{e}^{-\mathrm{i} n m T \Omega} & |m| \leq N \\ \hat{w}_{m}=T \sum_{n=-N}^{N} w_{n} \mathrm{e}^{-\mathrm{i} n m T \Omega} & |m| \leq N\end{cases}
$$

We can also rewrite the last equation in vector forms

$$
\underline{\hat{P}}=\underline{\underline{A}} \underline{P} \quad \text { and } \quad \underline{\hat{w}}=\underline{\underline{A}} \underline{w}
$$

where $\underline{P}, \underline{w}$ are column vectors of $P_{n}, w_{n}(-N \leq n \leq N)$ and $\underline{\underline{A}}$ is a square matrix defined from equation (28). Then, by substituting the last equation into the second equation of (20), we obtain

$$
\underline{\underline{A}} \underline{P}=\underline{\underline{K}} \underline{\underline{A}} \underline{w}+\underline{Q}
$$

where $\underline{Q}$ is a column vector of $Q_{n}=\mathcal{Q}_{T}(n \Omega)$ and $\underline{\underline{K}}$ is a diagonal matrix of $K_{n}=\mathcal{K}_{T}(n \Omega)+K_{s}(n \Omega)($ with $-N \leq n \leq N)$.

Finally, by combining equations (24) and (30), we obtain

$$
\underline{\underline{K}} \underline{\underline{A}} \underline{w}+\underline{\underline{A}} \underline{f}+\underline{Q}=0
$$

where $\underline{f}$ is a column vector of $f_{n}=f\left(w_{n}\right)$.

Equation (31) is nonlinear with regard to the unknown $\underline{w}$. If $f(w) \equiv 0$ (linear support), we can obtain the solution of this equation as follows

$$
\underline{w}_{0} \equiv-(\underline{\underline{K}} \underline{\underline{A}})^{-1} \underline{Q}=-\underline{\underline{A}}^{-1}\left(\underline{\underline{K}}^{-1} \underline{Q}\right)
$$


The last result is exactly the discretization of the analytic solution in equation (15). In a general case, we can use a numerical solver for equation (31). In this article, we use the solver "fsolve" of MATLAB ${ }^{\circledR}$. A difficulty is that this solver does not compute complex solutions. We need to decompose the unknown $\underline{w}$ into their real and imaginary parts. The initial value of the solver is taken by the linearised solution presented in equation (32).

\section{Example}

Let's consider a nonlinear support described by the constitutive law in equation (17) with $k_{s}=20 \mathrm{MN} / \mathrm{m}, \eta_{s}=0.1 \mathrm{MNs} / \mathrm{m}$ and the nonlinear law $f(w)=c w^{3}$ with $c=10 \mathrm{kN} / \mathrm{mm}^{3}$. We calculate the responses of a support by the numerical method in equation (31) with the parameters $N=250, T=0.001 \mathrm{~s}$ (thus, $\Omega=\pi / N T=4 \pi$ which corresponds to the frequency $2 \mathrm{~Hz}$ ). Figure [6
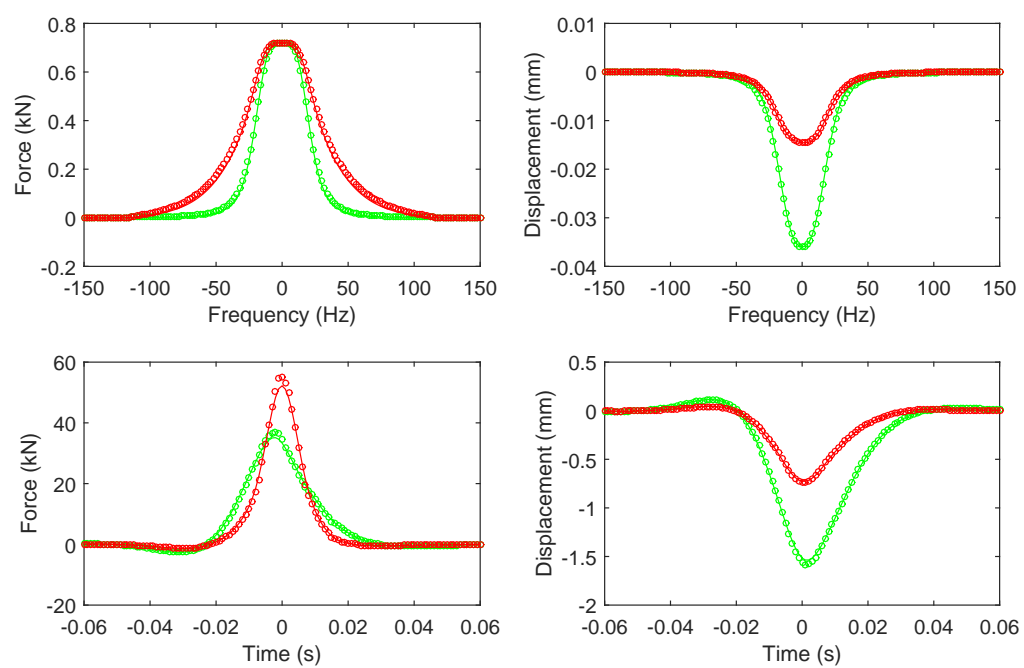

Figure 7: Responses of the supports with Timoshenko (circle) and Euler-Bernoulli (continuous line) beams with a linear (green) and a nonlinear (red) support behaviours

shows the results with the two beam models for linear and nonlinear behaviours. We see that the responses have similar forms for the two beams models and support behaviours. In particular, the reaction forces in the frequency domain have 
the same maximum pick at zero, but the nonlinear support has larger amplitudes in the high frequencies.

\section{Conclusion}

The system equivalence of a periodically supported beam has been developed for the Timoshenko beam and applied to nonlinear supports. This model has been compared with the existing model for an Euler-Bernoulli beam. Then, a numerical method has been developed for nonlinear supports based on the time-frequency transform which permits a fast calculation of the response of the support systems. Moreover, the numerical examples showed that the influence of the beam model is more important when the speed of the loads is higher and/or the support stiffness is larger.

\section{Acknowledgments}

This work has been developed in the context of a partnership between Eurotunnel Group and Ecole des Ponts ParisTech. The authors would like to thank the personnel of Eurotunnel Group for their support, specially J.L. Pochet, P. Joyez and R. Caby.

\section{Appendix A: Calculation of $\Psi(x, \omega)$ and $\Phi(x, \omega)$}

We will find the periodic solution of equation (7) by using the Fourier series developments of $\Phi$ and $\Psi$

$$
\Psi(x, \omega)=\sum_{n=-\infty}^{\infty} p_{n}(\omega) \mathrm{e}^{\mathrm{i} 2 \pi n \frac{x}{l}} \text { and } \Phi(x, \omega)=\sum_{n=-\infty}^{\infty} q_{n}(\omega) \mathrm{e}^{\mathrm{i} 2 \pi n \frac{x}{l}}
$$

with $p_{n}, q_{n}$ are computed by

$$
p_{n}(\omega)=\frac{1}{l} \int_{-l / 2}^{l / 2} \Psi(x, \omega) \mathrm{e}^{-\mathrm{i} 2 \pi n \frac{x}{l}} \mathrm{~d} x \quad \text { and } \quad q_{n}(\omega)=\frac{1}{l} \int_{-l / 2}^{l / 2} \Phi(x, \omega) \mathrm{e}^{-\mathrm{i} 2 \pi n \frac{x}{l}} \mathrm{~d} x
$$


It is remarkable that the Fourier coefficients of $\mathrm{e}^{\mathrm{i}} \frac{\omega}{v} x \hat{F}$ in equation (5) are calculated by

$\frac{1}{l} \int_{-l / 2}^{l / 2}\left[\hat{R}(\omega) \sum_{n=-\infty}^{\infty} \delta(x-n l)-\sum_{j=1}^{K} \frac{Q_{j}}{v} \mathrm{e}^{-\frac{\mathrm{i} \omega}{v} D_{j}}\right] \mathrm{e}^{-\mathrm{i} 2 \pi n \frac{x}{l}} \mathrm{~d} x=\frac{\hat{R}(\omega)}{l}-\frac{\delta_{0 n}}{v} \sum_{j=1}^{K} Q_{j} \mathrm{e}^{-\frac{\mathrm{i} \omega}{v} D_{j}}$

where $\delta_{0 n}=1$ if $n=0$ and $\delta_{0 n}=0$ if $n \neq 0$. Therefore, by performing the Fourier series development of equation (77) and by using the last equation, we obtain

$$
\left\{\begin{array}{l}
\kappa S G\left(\frac{i 2 \pi n}{l}-\frac{\mathrm{i} \omega}{v}\right) q_{n}=-\kappa S G\left(\frac{\omega}{v}-\frac{2 \pi n}{l}\right)^{2} p_{n}+\rho S \omega^{2} p_{n}+\frac{\hat{R}(\omega)}{l}-\frac{\delta_{0 n}}{v} \sum_{j=1}^{K} Q_{j} \mathrm{e}^{-\frac{\mathrm{i} \omega}{v} D_{j}} \\
\kappa S G\left(\frac{\mathrm{i} \omega}{v}-\frac{\mathrm{i} 2 \pi n}{l}\right) p_{n}=-E I\left(\frac{\omega}{v}-\frac{2 \pi n}{l}\right)^{2} q_{n}-\left(\kappa S G-\rho I \omega^{2}\right) q_{n}
\end{array}\right.
$$

Then, the following result is deduced from the second equation of (A4)

$$
q_{n}=\frac{-\kappa S G\left(\frac{\mathrm{i} \omega}{v}-\frac{\mathrm{i} 2 \pi n}{l}\right) p_{n}}{E I\left(\frac{\omega}{v}-\frac{2 \pi n}{l}\right)^{2}+\left(\kappa S G-\rho I \omega^{2}\right)}
$$

Then, by substituting the last equation into equation (A4), we obtain

$$
\left\{\begin{array}{l}
p_{n}=\tilde{p}_{n} \hat{R}(\omega)-\delta_{0 n} \frac{\tilde{p}_{0} l}{v} \sum_{j=1}^{K} Q_{j} \mathrm{e}^{-\frac{\mathrm{i} \omega}{v} D_{j}} \\
q_{n}=\tilde{q}_{n} \hat{R}(\omega)-\delta_{0 n} \frac{\tilde{q}_{0} l}{v} \sum_{j=1}^{K} Q_{j} \mathrm{e}^{-\frac{\mathrm{i} \omega}{v} D_{j}}
\end{array}\right.
$$

where $\tilde{p}_{n}, \tilde{q}_{n}$ are calculated by

$$
\left\{\begin{array}{l}
\tilde{p}_{n}=\frac{\kappa S G-\rho I \omega^{2}+E I\left(\frac{\omega}{v}-\frac{2 \pi n}{l}\right)^{2}}{l z_{n}} \\
\tilde{q}_{n}=-\frac{i \kappa S G\left(\frac{\omega}{v}-\frac{2 \pi n}{l}\right)}{l z_{n}}
\end{array}\right.
$$

with

$$
z_{n}=\kappa S G E I\left(\frac{\omega}{v}-\frac{2 \pi n}{l}\right)^{4}-\rho I S \omega^{2}(\kappa G+E)\left(\frac{\omega}{v}-\frac{2 \pi n}{l}\right)^{2}+\rho S \omega^{2}\left(\rho I \omega^{2}-\kappa S G\right)
$$

By substituting equation (A6) into equation (A1), we obtain:

$$
\left\{\begin{array}{l}
\Phi(x, \omega)=\hat{R}(\omega) \sum_{n=-\infty}^{\infty} \tilde{p}_{n} \mathrm{e}^{\mathrm{i} 2 \pi n \frac{x}{l}}-\tilde{p}_{0} \frac{l}{v} \sum_{j=1}^{K} Q_{j} \mathrm{e}^{-\frac{\mathrm{i} \omega}{v} D_{j}} \\
\Psi(x, \omega)=\hat{R}(\omega) \sum_{n=-\infty}^{\infty} \tilde{q}_{n} \mathrm{e}^{\mathrm{i} 2 \pi n \frac{x}{l}}-\tilde{q}_{0} \frac{l}{v} \sum_{j=1}^{K} Q_{j} \mathrm{e}^{-\frac{\mathrm{i} \omega}{v} D_{j}}
\end{array}\right.
$$


Remark: We can obtain the expressions of $\tilde{p}_{0}, \tilde{q}_{0}$ by substituting $n=0$ into equation (A7)

$$
\left\{\begin{array}{l}
\tilde{p}_{0}=\frac{\kappa S G-\rho I \omega^{2}+E I \frac{\omega^{2}}{v^{2}}}{l\left[\kappa S G\left(E I \frac{\omega^{4}}{v^{4}}-\rho S \omega^{2}\right)-\rho S I\left(\kappa G+E-\rho v^{2}\right) \frac{\omega^{4}}{v^{2}}\right]} \\
\tilde{q}_{0}=\frac{-\mathrm{i} \kappa S G \frac{\omega}{v}}{l\left[\kappa S G\left(E I \frac{\omega^{4}}{v^{4}}-\rho S \omega^{2}\right)-\rho S I\left(\kappa G+E-\rho v^{2}\right) \frac{\omega^{4}}{v^{2}}\right]}
\end{array}\right.
$$

\section{Appendix B: Analytical expressions of $\eta(x, \omega)$ and $\gamma(x, \omega)$}

The function $z_{n}$ in equation (A8) is a second-order polynomial with regard to $\left(\frac{\omega}{v}-\frac{2 \pi n}{L}\right)^{2}$ and we can rewrite it as follows

$$
z_{n}(\omega)=\kappa S G E I\left[\left(\frac{\omega}{v}-\frac{2 \pi n}{l}\right)^{2}-\lambda_{1}^{2}\right]\left[\left(\frac{\omega}{v}-\frac{2 \pi n}{l}\right)^{2}+\lambda_{2}^{2}\right]
$$

where $\lambda_{1}, \lambda_{2}$ are given by

$$
\left\{\begin{array}{l}
\lambda_{1}^{2}=\sqrt{\frac{\omega^{4}}{4}\left(\frac{\rho}{E}-\frac{\rho}{\kappa G}\right)^{2}+\frac{\rho S \omega^{2}}{E I}}+\frac{\omega^{2}}{2}\left(\frac{\rho}{E}+\frac{\rho}{\kappa G}\right) \\
\lambda_{2}^{2}=\sqrt{\frac{\omega^{4}}{4}\left(\frac{\rho}{E}-\frac{\rho}{\kappa G}\right)^{2}+\frac{\rho S \omega^{2}}{E I}}-\frac{\omega^{2}}{2}\left(\frac{\rho}{E}+\frac{\rho}{\kappa G}\right)
\end{array}\right.
$$

In order to simplify the expression of $\tilde{p}_{n}$, we substitute equation (B1) into the first equation of (A7), we can write

$$
\tilde{p}_{n}(\omega)=\frac{1}{l E I\left(\lambda_{1}^{2}+\lambda_{2}^{2}\right)}\left[\frac{C_{1}}{\left(\frac{\omega}{v}-\frac{2 \pi n}{l}\right)^{2}-\lambda_{1}^{2}}-\frac{C_{2}}{\left(\frac{\omega}{v}-\frac{2 \pi n}{l}\right)^{2}+\lambda_{2}^{2}}\right]
$$

Then, by taking the equality of the coefficients of two expression of $\tilde{p}_{n}$ in equations (A7) and (B3), we obtain

$$
\left\{\begin{array}{l}
C_{1}=1-\frac{\rho I \omega^{2}-E I \lambda_{1}^{2}}{\kappa S G} \\
C_{2}=1-\frac{\rho I \omega^{2}+E I \lambda_{2}^{2}}{\kappa S G}
\end{array}\right.
$$

Similarly, we have

$$
\tilde{q}_{n}(\omega)=\frac{-\mathrm{i}}{l E I\left(\lambda_{1}^{2}+\lambda_{2}^{2}\right)}\left[\frac{\frac{\omega}{v}-\frac{2 \pi n}{l}}{\left(\frac{\omega}{v}-\frac{2 \pi n}{l}\right)^{2}-\lambda_{1}^{2}}-\frac{\frac{\omega}{v}-\frac{2 \pi n}{l}}{\left(\frac{\omega}{v}-\frac{2 \pi n}{l}\right)^{2}+\lambda_{2}^{2}}\right]
$$

In order to deduce the analytical expression of $\eta(x, \omega)$ in equations (9), we need to calculate the infinite sum of the series which are presented in equation (B5). Let's consider the following function

$$
f(x)=\frac{\sin \lambda_{1}(l-x)+\mathrm{e}^{-\mathrm{i} \frac{\omega l}{v}} \sin \lambda_{1} x}{\cos l \lambda_{1}-\cos \frac{\omega l}{v}} \mathrm{e}^{\mathrm{i} \frac{\omega}{v} x} \quad \forall x \in[0, l]
$$


Because $f(0)=f(l)$, the Fourier coefficients of this function are calculated by

$$
c_{n}=\frac{1}{l} \int_{0}^{l} f(x) \mathrm{e}^{-\mathrm{i} \frac{2 \pi n x}{l}} \mathrm{~d} x=\frac{2}{l} \frac{\lambda_{1}}{\left(\frac{\omega}{v}-\frac{2 \pi n}{l}\right)^{2}-\lambda_{1}^{2}}
$$

We can write this function in the Fourier series expansion as follows

$$
f(x)=\sum_{n=-\infty}^{\infty} c_{n} \mathrm{e}^{\mathrm{i} \frac{2 \pi n x}{l}}
$$

By substituting equations (B6), (B7) into the last equation, we obtain

$$
\frac{\sin \lambda_{1}(l-x)+\mathrm{e}^{-\mathrm{i} \frac{\omega}{v}} \sin \lambda_{1} x}{\cos l \lambda_{1}-\cos \frac{\omega l}{v}}=\frac{2}{l} \sum_{n=-\infty}^{\infty} \frac{\lambda_{1} \mathrm{e}^{\mathrm{i} x\left(\frac{2 \pi n}{l}-\frac{\omega}{v}\right)}}{\left(\frac{\omega}{v}-\frac{2 \pi n}{l}\right)^{2}-\lambda_{1}^{2}}
$$

Similarly, we have

$$
\frac{\sinh \lambda_{2}(l-x)+\mathrm{e}^{-\mathrm{i} \frac{\omega l}{v}} \sinh \lambda_{2} x}{\cosh l \lambda_{2}-\cos \frac{\omega l}{v}}=\frac{2}{l} \sum_{n=-\infty}^{\infty} \frac{\lambda_{2} \mathrm{e}^{\mathrm{i} x\left(\frac{2 \pi n}{l}-\frac{\omega}{v}\right)}}{\left(\frac{\omega}{v}-\frac{2 \pi n}{l}\right)^{2}+\lambda_{2}^{2}}
$$

Finally, the analytical expression of $\eta(x, \omega)$ in equations (9) can be obtained by combining (B3) and the two last results

$$
\begin{array}{r}
\eta(x, \omega)=\frac{1}{2 E I\left(\lambda_{1}^{2}+\lambda_{2}^{2}\right)}\left[\frac{C_{1}}{\lambda_{1}} \frac{\sin \lambda_{1}(l-x)+\mathrm{e}^{-\mathrm{i} \frac{\omega l}{v}} \sin \lambda_{1} x}{\cos l \lambda_{1}-\cos \frac{\omega l}{v}}\right. \\
\left.-\frac{C_{2}}{\lambda_{2}} \frac{\sinh \lambda_{2}(l-x)+\mathrm{e}^{-\mathrm{i} \frac{\omega l}{v}} \sinh \lambda_{2} x}{\cosh l \lambda_{2}-\cos \frac{\omega l}{v}}\right]
\end{array}
$$

In the similar way, we can deduce the analytic expression of $\gamma(x, \omega)$ by applying the derivation with regard to $x$ of equations (B9) and (B10)

$$
\begin{gathered}
\frac{\cos \lambda_{1}(l-x)-\mathrm{e}^{-\mathrm{i} \frac{\omega l}{v}} \cos \lambda_{1} x}{\cos l \lambda_{1}-\cos \frac{\omega l}{v}}=\frac{2}{l} \sum_{n=-\infty}^{\infty} \frac{\mathrm{i}\left(\frac{2 \pi n}{l}-\frac{\omega}{v}\right) \mathrm{e}^{\mathrm{i} x\left(\frac{2 \pi n}{l}-\frac{\omega}{v}\right)}}{\left(\frac{\omega}{v}-\frac{2 \pi n}{l}\right)^{2}-\lambda_{1}^{2}} \\
\frac{\cosh \lambda_{2}(l-x)-\mathrm{e}^{-\mathrm{i} \frac{\omega l}{v}} \cosh \lambda_{2} x}{\cosh l \lambda_{2}-\cos \frac{\omega l}{v}}=\frac{2}{l} \sum_{n=-\infty}^{\infty} \frac{\mathrm{i}\left(\frac{2 \pi n}{l}-\frac{\omega}{v}\right) \mathrm{e}^{\mathrm{i} x\left(\frac{2 \pi n}{l}-\frac{\omega}{v}\right)}}{\left(\frac{\omega}{v}-\frac{2 \pi n}{l}\right)^{2}+\lambda_{2}^{2}}
\end{gathered}
$$

Then, by combining the two last equations and equations (B4), we obtain the analytical expression of $\gamma(x, \omega)$ in equation (9)

$$
\begin{array}{r}
\gamma(x, \omega)=\frac{\mathrm{i}}{2 E I\left(\lambda_{1}^{2}+\lambda_{2}^{2}\right)}\left[\frac{\cos \lambda_{1}(l-x)-\mathrm{e}^{-\mathrm{i} \frac{\omega l}{v}} \cos \lambda_{1} x}{\cos l \lambda_{1}-\cos \frac{\omega l}{v}}\right. \\
\left.-\frac{\cosh \lambda_{2}(l-x)-\mathrm{e}^{-\mathrm{i} \frac{\omega l}{v}} \cosh \lambda_{2} x}{\cosh l \lambda_{2}-\cos \frac{\omega l}{v}}\right]
\end{array}
$$


Remark: We obtain the expression of $\eta(0, \omega)$ by substituting $x=0$ into equation (B11)

$$
\eta(0, \omega)=\frac{1}{2 E I\left(\lambda_{1}^{2}+\lambda_{2}^{2}\right)}\left[\frac{C_{1}}{\lambda_{1}} \frac{\sin \lambda_{1} l}{\cos l \lambda_{1}-\cos \frac{\omega l}{v}}-\frac{C_{2}}{\lambda_{2}} \frac{\sinh \lambda_{2} l}{\cosh l \lambda_{2}-\cos \frac{\omega l}{v}}\right]
$$

\section{References}

[1] D. J. Mead, Free wave propagation in periodically supported, infinite beams, Journal of Sound and Vibration 11 (2) (1970) 181-197.

[2] D. J. Mead, Wave propagation in continuous periodic structures: research contributions from southampton, Journal of Sound and Vibration 190 (3) (1996) 495-524.

[3] A. V. Metrikine, K. Popp, Vibration of a periodically supported beam on an elastic half-space, European Journal of Mechanics A/Solid 18 (1999) 679-701.

[4] A. V. Vostroukhov, A. Metrikine, Periodically supported beam on a viscoelastic layer as a model for dynamic analysis of a high-speed railway track, International Journal of Solids and Structures 40 (2003) 5723-5752.

[5] P. M. Belotserkovskiy, On the oscillation of infinite periodic beams subjected to a moving concentrated force, Journal of Sound and Vibration 193 (3) (1996) 705-712.

[6] X. Sheng, C. Jones, D. Thompson, Response of infinite periodic structure to moving or stationary harmonic loads, Journal of Sound and Vibration 282 (2005) 125-149.

[7] X. Sheng, M. Li, C. J. C. Jones, D. J. Thompson, Vibration analysis of the continuous beam subjected to a moving mass, Journal of Sound and Vibration 303 (2007) 873-894.

[8] A. Nordborg, Vertical rail vibrations: Pointforce excitation, Acustica 84 (1998) 280-288. 
[9] A. Nordborg, Vertical rail vibrations: Parametric excitation, Acustica 84 (1998) 289-300.

[10] J.-F. Hamet, Railway noise: Use of the timoshenko model in rail vibration studies, Acustica 85 (1999) 54-62.

[11] T. Hoang, D. Duhamel, G. Foret, H. Yin, P. Joyez, R. Caby, Dynamical response of railway tracks in tunnel, $11^{\text {th }}$ World Congress on Computational Mechanics (WCCM XI), Barcelona, Spain, July 20-25, 2014.

[12] M. Ansari, E. Esmailzadeh, D. Younesian, Frequency analysis of finite beams on nonlinear KelvinVoight foundation under moving loads, Journal of Sound and Vibration 330 (7) (2011) 1455-1471.

[13] M. Kargarnovin, D. Younesian, D. Thompson, C. Jones, Response of beams on nonlinear viscoelastic foundations to harmonic moving loads, Computers \& Structures 83 (23-24) (2005) 1865-1877.

[14] H. Ding, K. L. Shi, L. Q. Chen, S. P. Yang, Dynamic response of an infinite Timoshenko beam on a nonlinear viscoelastic foundation to a moving load, Nonlinear Dynamics 73 (1-2) (2013) 285-298.

[15] H. Ding, L.-Q. Chen, S.-P. Yang, Convergence of Galerkin truncation for dynamic response of finite beams on nonlinear foundations under a moving load, Journal of Sound and Vibration 331 (10) (2012) 2426-2442.

[16] J.-S. Chen, Y.-K. Chen, Steady state and stability of a beam on a damped tensionless foundation under a moving load, International Journal of NonLinear Mechanics 46 (1) (2011) 180-185.

[17] E. Sapountzakis, A. Kampitsis, Nonlinear response of shear deformable beams on tensionless nonlinear viscoelastic foundation under moving loads, Journal of Sound and Vibration 330 (22) (2011) 5410-5426.

[18] W. Walter, Ordinary differential equations, Springer, 1998.

[19] S. Mallat, A Wavelet Tour of Signal Processing, Academic Press, 2009. 